\title{
An IGISOL portrait - Selected contributions
}

Published online: 18 April 2012 - (C) Società Italiana di Fisica / Springer-Verlag 2012

This special issue of the European Physical Journal A consists of ten articles describing selected scientific work related to the research at the on-line isotope separator facility IGISOL at the University of Jyväskylä, Finland.

Nuclear-decay and ground-state spectroscopy experienced a promising step forward in the early 1980s when the Ion Guide Isotope Separator On-Line (IGISOL) concept was brought into operation at the $20 \mathrm{MeV}$ proton cyclotron of the Physics Department of the University of Jyväskylä (JYFL). For the first time, it became possible to separate short-lived nuclides with ms delay times by using an ISOL technique unrestricted by chemistry. Access to short-lived isotopes of refractory elements, thus, opened up new regions in the nuclear chart for ISOL-based spectroscopy.

During the first decade of its operation the main research activity at IGISOL concentrated on decay spectroscopy, and, in particular, on the fission products produced in the proton-induced fission of ${ }^{238} \mathrm{U}$. About 40 new isotope and isomer decays were discovered and studied employing beta, gamma, and conversion electron spectroscopy. These experiments covered a broad range of neutron-rich nuclei from yttrium $(Z=39)$ to cadmium $(Z=48)$ and revealed how their structure was developing between highly deformed Zr through triaxial Ru isotopes to weakly deformed vibrational $\mathrm{Cd}$ isotopes.

The original IGISOL facility was shut down in October 1991 and was reinstalled as IGISOL 2 at the science campus housing the laboratory for the $K=130 \mathrm{MeV}$ heavy-ion cyclotron. A new window of opportunity for extending the research to neutron-deficient nuclei far from stability was opened and was going to have strong impact in extending the scope of the IGISOL research program. The first two decades of IGISOL were reviewed in Nuclear Physics A 693, 477 (2001).

A major leap forward was experienced in the last decade when the front end of the separator was upgraded and novel ion trap installations were introduced. Thus the IGISOL 3 facility came into existence. This enabled new perspectives for precision experiments on both neutron-rich fission products and neutron-deficient nuclei produced in light- and heavy-ion fusion reactions. The experiments covered both decay spectroscopy and accurate measurements of ground-state properties such as charge radii and masses. A crucial aspect in these studies is the use of cooled and bunched beams in collinear laser spectroscopy and Penning-trap experiments.

Currently, the facility is again being upgraded and reassembled in a new accelerator hall, served by two cyclotron driver accelerators, the K130 heavy-ion cyclotron and a high-intensity $30 \mathrm{MeV}$ proton cyclotron MCC30. Intense lightion beams make possible the use of both fusion and fission reactions providing access to light proton-rich nuclei and an extended range of neutron-rich nuclei produced in fission. IGISOL 4 will come online in 2012.

This issue of the European Physical Journal A presents articles related to recent scientific developments at the IGISOL facility. The selected articles include recent original and earlier unpublished results which cover aspects of the scientific research related to the current scientific profile of IGISOL. These articles are papers selected from the main volume of the IGISOL Portrait to be published by Springer. 